\title{
CHARACTERIZATIONS OF SEMIPERFECT AND PERFECT RINGS $\left.{ }^{*}\right)$
}

\author{
Weimin Xue
}

\begin{abstract}
We characterize semiperfect modules, semiperfect rings, and perfect rings using locally projective covers and generalized locally projective covers, where locally projective modules were introduced by Zimmermann-Huisgen and generalized locally projective coves are adapted from Azumaya's generalized projective covers.
\end{abstract}

\section{Introduction}

Azumaya [A2] introduced the notion of generalized projective covers to characterize semiperfect modules and rings. Adapting his concept, we call a module epimorphism $f: P \longrightarrow M$ a (generalized) cover in case $(\operatorname{Ker}(f) \subseteq \operatorname{Rad}(P)) \operatorname{Ker}(f) \ll P$. A (generalized) cover $f: P \longrightarrow M$ is called a (generalized) projective cover in case $P$ is a projective module, and it is called a (generalized) locally projective cover in case $P$ is a locally projective module.

This paper consists of three sections. We obtain some basic properties of (generalized) covers in Section 1. In Section 2, we characterize (generalized) semiperfect modules via (generalized) projective covers of the (generalized) complements. In Section 3, we characterize semiperfect rings and modules, perfect rings, and quasi-perfect rings $[\mathbf{C X}]$, using (generalized) locally projective covers.

The terminologies and notations of Anderson and Fuller $[\mathbf{A F}]$ will be freely used. We refer the reader to [AF, Section 27, Section 28] for a presentation of semiperfect and perfect rings. Throughout $R$ is an associative ring with identity whose Jacobson radical is denoted by $J$. Unless otherwise stated, modules are unitary left $R$-modules, and homomorphisms are left $R$-module homomorphisms. If $M$ is a module, we recall from $[\mathbf{A F}]$ that $\operatorname{Rad}(M)$ denotes the radical of $M$, and $(U \ll$ $M) U \leq M$ means that $U$ is a (superfluous) submodule of $M$.

${ }^{(*)}$ This research is supported by the National Natural Science Foundation of China. 


\section{Basic properties of (generalized) covers}

If $P$ and $M$ are modules, we call an epimorphism $f: P \longrightarrow M$ a (generalized) cover in case $(\operatorname{Ker}(f) \subseteq \operatorname{Rad}(P)) \operatorname{Ker}(f) \ll P$. Since $\operatorname{Rad}(P)$ is the sum of all superfluous submodules of $P$, every cover is a generalized cover. We have the following basic properties of (generalized) covers.

Lemma 1.1. If both $f: P \longrightarrow M$ and $g: M \longrightarrow N$ are (generalized) covers, then $g f: P \longrightarrow N$ is a (generalized) cover.

Proof: If both $f$ and $g$ are covers, then $g f$ is a cover by [AF, Proposition 5.17(1)].

Now let both $f$ and $g$ be generalized covers. To show $\operatorname{Ker}(g f) \subseteq$ $\operatorname{Rad}(P)$, we let $p \in \operatorname{Ker}(g f)$. Then $g f(p)=0$ and $f(p) \subseteq \operatorname{Ker}(g) \subseteq$ $\operatorname{Rad}(M)$. Since $\operatorname{Ker}(f) \subseteq \operatorname{Rad}(P)$, it follows from [AF, Proposition 9.15] that $f(\operatorname{Rad}(P))=\operatorname{Rad}(M)$. Hence $f(p)=f\left(p^{\prime}\right)$ for some $p^{\prime} \in \operatorname{Rad}(P)$, so $p-p^{\prime} \in \operatorname{Ker}(f) \subseteq \operatorname{Rad}(P)$. We obtain $p \in \operatorname{Rad}(P)$.

Lemma 1.2. (1) If each $f_{i}: P_{i} \longrightarrow M_{i}(i=1,2, \ldots, n)$ is a cover then $\oplus_{i=1}^{n} f_{i}: \oplus_{i=1}^{n} P_{i} \longrightarrow \oplus_{i=1}^{n} M_{i}$ is a cover.

(2) If each $f_{i}: P_{i} \longrightarrow M_{i}(i \in I)$ is a generalized cover then $\oplus_{i \in I} f_{i}$ : $\oplus_{i \in I} P_{i} \longrightarrow \oplus_{i \in I} M_{i}$ is a generalized cover.

Proof: (1) Since each $\operatorname{Ker}\left(f_{i}\right) \ll P_{i}$ we have $\operatorname{Ker}\left(\oplus_{i=1}^{n} f_{i}\right)=$ $\oplus_{i=1}^{n} \operatorname{Ker}\left(f_{i}\right) \ll \oplus_{i=1}^{n} P_{i}$. So $\oplus_{i=1}^{n} f_{i}$ is a cover.

(2) Since each $\operatorname{Ker}\left(f_{i}\right) \subseteq \operatorname{Rad}\left(P_{i}\right)$ we have $\operatorname{Ker}\left(\oplus_{i \in I} f_{i}\right)=\oplus_{i \in I} \operatorname{Ker}\left(f_{i}\right) \subseteq$ $\oplus_{i \in I} \operatorname{Rad}\left(P_{i}\right)=\operatorname{Rad}\left(\oplus_{i \in I} P_{i}\right)$. So $\oplus_{i \in I} f_{i}$ is a generalized cover.

Lemma 1.3. Let $f: P \longrightarrow M$ be a cover. If $M$ is finitely generated then $P$ is also finitely generated.

Proof: Since $P / \operatorname{Ker}(f) \cong M$ is finitely generated, there is a finitely generated submodule $P^{\prime}$ of $P$ such that

$$
P^{\prime}+\operatorname{Ker}(f)=P .
$$

Since $\operatorname{Ker}(f) \ll P$ we have $P^{\prime}=P$. 


\section{2. (Generalized) projective covers and $M$-projective covers}

Let $M$ be a module. If $U, U^{\prime} \leq M$ and $M=U+U^{\prime}$ then $U^{\prime}$ is called a (generalized) complement of $U$ in case $\left(U \cap U^{\prime} \subseteq \operatorname{Rad}\left(U^{\prime}\right)\right) U \cap U^{\prime} \ll U^{\prime}$. Clearly, each complement is a generalized complement.

A (generalized) cover $f: P \longrightarrow M$ is called a (generalized) projective cover in case $P$ is a projective module. Since every cover is a generalized cover, a projective cover is a generalized projective cover as observed in [A2].

A connection between (generalized) projective covers and (generalized) complements is given as follows.

Proposition 2.1. If $M$ is a module and $U \leq M$, then the following three statements are equivalent.

(1) $M / U$ has a (generalized) projective cover.

(2) If $V \leq M$ and $M=U+V$ then $U$ has a (generalized) complement $U^{\prime} \subseteq V$ such that $U^{\prime}$ has a (generalized) projective cover.

(3) $U$ has a (generalized) complement $U^{\prime}$ which has a (generalized) projective cover.

Proof: $(1) \Rightarrow(2)$. Let $f: P \longrightarrow M / U$ be a (generalized) projective cover. Since $M=U+V$,

$$
\begin{aligned}
g: V & \longrightarrow M / V \text { via } \\
v & \longmapsto v+U
\end{aligned}
$$

is an epimorphism. Since $P$ is projective, there is a homomorphism $h: P \longrightarrow V$ such that $f=g h$. It is easy to see that $M=U+h(P)$ where $h(P) \subseteq V$. Now $(\operatorname{Ker}(f) \subseteq \operatorname{Rad}(P)) \operatorname{Ker}(f) \ll P$, so we have

$$
U \cap h(P)=h(\operatorname{Ker}(f))(\subseteq h(\operatorname{Rad}(P)) \subseteq \operatorname{Rad}(h(P))) \ll h(P)
$$

and $h(P)$ is a (generalized) complement of $U \subseteq V$. Since $\operatorname{Ker}(h) \subseteq$ $\operatorname{Ker}(f)(\subseteq \operatorname{Rad}(P)) \ll P$,

$$
h: P \longrightarrow h(P)
$$

is a (generalized) projective cover.

$(2) \Rightarrow(3)$. This is obvious.

$(3) \Rightarrow(1)$. Let $f: P \longrightarrow U^{\prime}$ be a (generalized) projective cover. Since $U^{\prime}$ is a (generalized) complement of $U$, the natural epimorphism

$$
g: U^{\prime} \longrightarrow U^{\prime} /\left(U \cap U^{\prime}\right) \stackrel{h}{\cong}\left(U+U^{\prime}\right) / U=M / U
$$


is a (generalized) cover. Hence $h g f: P \longrightarrow M / U$ is a (generalized) projective cover by Lemma 1.1.

Let $M$ be a module. $M$ is called (generalized) complemented in case each submodule $U$ has a (generalized) complement $U^{\prime}$, and it is called (generalized) amply complemented in case $M=U+V$ implies that $U$ has a (generalized) complement $U^{\prime} \subseteq V$. According to [A2], $M$ is called (generalized) semiperfect in case each factor module of $M$ has a (generalized) projective cover. Azumaya [A2, Theorem 4] proved that $M$ is generalized semiperfect if and only if each proper submodule of $M$ is contained in a maximal submodule of $M$ and each simple factor module of $M$ has a generalized projective cover. The next different characterization of (generalized) semiperfect modules follows immediately from Proposition 2.1, where the non-parenthetical version is [F, Theorem 1]. A characterization of semiperfect modules using locally projective covers will be given in the next section.

Theorem 2.2. The following three statements are equivalent for a module $M$.

(1) $M$ is (generalized) semiperfect.

(2) $M$ is (generalized) amply complemented by complements which have (generalized) projective covers.

(3) $M$ is (generalized) complemented by complements which have (generalized) projective covers.

A (generalized) cover $f: P \longrightarrow M$ is called a (generalized) $M$ projective cover in case $P$ is a $M$-projective module. Modifying the proof of Proposition 2.1, we have an analogous result.

Proposition 2.3. If $M$ is a module and $U \leq M$, then the following three statements are equivalent.

(1) $M / U$ has a (generalized) $M$-projective cover.

(2) If $V \leq M$ and $M=U+V$ then $U$ has a (generalized) complement $U^{\prime} \subseteq V$ such that $U^{\prime}$ has a (generalized) $M$-projective cover.

(3) $U$ has a (generalized) complement $U^{\prime}$ which has a (generalized) $M$-projective cover.

We call a module $M$ (generalized) quasi-semiperfect in case each factor module of $M$ has a (generalized) $M$-projective cover. Now we have the following result by Proposition 2.3.

Theorem 2.4. The following three statements are equivalent for a module $M$.

(1) $M$ is (generalized) quasi-semiperfect. 
(2) $M$ is (generalized) amply complemented by complements which have (generalized) $M$-projective covers.

(3) $M$ is (generalized) complemented by complements which have (generalized) $M$-projective covers.

Using an idea of Azumaya's proof given in [A2, Theorem 4] we obtain

Theorem 2.5. Let $R$ be a semilocal ring and $M$ a finitely generated left $R$-module. Then $M$ is (generalized) quasi-semiperfect if and only if each simple factor module of $M$ has a (generalized) $M$-projective cover.

Proof: $(\Rightarrow)$. This is obvious.

$(\Leftarrow)$. Let $U \leq M$ and $\bar{M}=M / U$. Since $R$ is semilocal and $\bar{M}$ is finitely generated, $J \bar{M}=\operatorname{Rad}(\bar{M}) \ll \bar{M}$ and $\bar{M} / J \bar{M}$ is semisimple. Let $\bar{M} / J \bar{M}=\oplus_{i=1}^{n} S_{i}$ be a direct sum of simple submodules $S_{i}$ $(i=1,2, \ldots, n)$. Since each $S_{i}$ is isomorphic to a simple factor module of $M$, it has a (generalized) $M$-projective cover $f_{i}: P_{i} \longrightarrow S_{i}$ where $\left(\operatorname{Ker}\left(f_{i}\right) \subseteq \operatorname{Rad}\left(P_{i}\right)=J P_{i}\right) \operatorname{Ker}\left(f_{i}\right) \ll P_{i}$. Since $P_{i} / \operatorname{Ker}\left(f_{i}\right) \cong S_{i}$ is simple, $\operatorname{Ker}\left(f_{i}\right)$ is a maximal submodule of $P$ and so $\left(\operatorname{Ker}\left(f_{i}\right)=J P_{i}\right)$ $\operatorname{Ker}\left(f_{i}\right)=J P_{i} \ll P_{i}$. By Lemma 1.2,

$$
f=\oplus_{i=1}^{n} f_{i}: P=\oplus_{i=1}^{n} P_{i} \longrightarrow \oplus_{i=1}^{n} S_{i}=\bar{M} / J \bar{M}
$$

is a (generalized) $M$-projective cover, where $(\operatorname{Ker}(f)=J P) \operatorname{Ker}(f)=$ $J P \ll P$. Let $g: \bar{M} \longrightarrow \bar{M} / J \bar{M}$ be the natural epimorphism. Since $P$ is $M$-projective, there is a homomorphism $h: P \longrightarrow \bar{M}$ such that $f=g h$. Now $f$ is an epimorphism and $\operatorname{Ker}(g)=J \bar{M} \ll \bar{M}$, it follows from $[\mathbf{A F}$, Corollary 5.15] that $h$ is an epimorphism. Since $(\operatorname{Ker}(h) \subseteq \operatorname{Ker}(f)=J P)$ $\operatorname{Ker}(h) \subseteq \operatorname{Ker}(f)=J P \ll P$, we see that

$$
h: P \longrightarrow \bar{M}
$$

is a (generalized) $M$-projective cover. Hence $M$ is a (generalized) quasisemiperfect module.

\section{3. (Generalized) locally projective covers}

A module $P$ is called locally projective $[\mathbf{Z 1}]$ in case it satisfies any of the following equivalent conditions: (a) if $A$ and $B$ are modules, $g: A \longrightarrow B$ is an epimorphism and $f: P \longrightarrow B$ is a homomorphism then for every finitely generated (cyclic) submodule $P_{0}$ of $P$ there is a homomorphism $h: P \longrightarrow A$ such that $\left.f\right|_{P_{0}}=\left.g h\right|_{P_{0}} ;(\mathrm{b})$ if $M$ is a module and $f: M \longrightarrow$ 
$P$ is an epimorphism then for every finitely generated (cyclic) submodule $P_{0}$ of $P$ there is a homomorphism $g: P \longrightarrow M$ such that $\left.f g\right|_{P_{0}}=1_{P_{0}}$. Clearly, every finitely generated (even countably generated [A1]) locally projective module is projective. The following facts are also known and we shall freely use them without reference (for the proofs, see $[\mathbf{Z 1}]$ and [A1]): (1) a direct sum of modules is locally projective if and only if each summand is locally projective; (2) a pure submodule of a locally projective module is locally projective; and (3) if $P$ is a locally projective module, then (i) $P$ is flat, (ii) $\operatorname{Rad}(P)=J P$, and (iii) if $\operatorname{Rad}(P)=P$ then $P=0$.

A (generalized) cover $f: P \longrightarrow M$ is called a (generalized) locally projective cover in case $P$ is a locally projective module. Since any cover is a generalized cover, a locally projective cover is a generalized locally projective cover. According to the facts of locally projective modules and Lemmas 1.1, 1.2 and 1.3, we have the following three lemmas.

Lemma 3.1. If $f: P \longrightarrow M$ is a (generalized) locally projective cover and $g: M \longrightarrow N$ is a (generalized) cover then $g f: P \longrightarrow N$ is a (generalized) locally projective cover.

Lemma 3.2. (1) If each $f_{i}: P_{i} \longrightarrow M_{i}(i=1,2, \ldots, n)$ is a locally projective cover then $\oplus_{i=1}^{n} f_{i}: \oplus_{i=1}^{n} P_{i} \longrightarrow \oplus_{i=1}^{n} M_{i}$ is a locally projective cover.

(2) If each $f_{i}: P_{i} \longrightarrow M_{i}(i \in I)$ is a generalized locally projective cover then $\oplus_{i \in I} f_{i}: \oplus_{i \in I} P_{i} \longrightarrow \oplus_{i \in I} M_{i}$ is a generalized locally projective cover.

Lemma 3.3. Let $f: P \longrightarrow M$ be a locally projective cover. If $M$ is a finitely generated module then $P$ is a finitely generated projective module.

The following proposition is an analogous result of [A2, Proposition 1].

Proposition 3.4. Let $f: P \longrightarrow M$ be a generalized locally projective cover. If $g: Q \longrightarrow M$ is a projective cover where $Q$ is finitely generated then there is an isomorphism $h: P \cong Q$ such that $f=g h$.

Proof: Let $Q=\sum_{i=1}^{n} R q_{i}$. Then $f\left(p_{i}\right)=g\left(q_{i}\right)$ for some $p_{i} \in P$ $(i=1,2, \ldots, n)$. Since $P_{0}=\sum_{i=1}^{n} R p_{i}$ is a finitely generated submodule of $P$ there is a homomorphism $h: P \longrightarrow Q$ such that $\left.f\right|_{P_{0}}=\left.g h\right|_{P_{0}}$. Now $g\left(q_{i}\right)=f\left(p_{i}\right)=g h\left(p_{i}\right)$, so $q_{i}-h\left(p_{i}\right) \in \operatorname{Ker}(g)$ and we have $Q=$ $h\left(P_{0}\right)+\operatorname{Ker}(g)$. But $\operatorname{Ker}(g) \ll Q$ we obtain $h\left(P_{0}\right)=Q$, so $h(P)=Q$ and $h$ is an epimorphism. Since $Q$ is projective, $h$ splits. Let $K=\operatorname{Ker}(h) \subseteq$ $\operatorname{Ker}(f)$ and $P=K \oplus K^{\prime}$ for some $K^{\prime} \leq P$. Since $f: P \longrightarrow M$ is 
a generalized locally projective cover, we have $\operatorname{Ker}(f) \subseteq J P$ and then $K \subseteq J P=J K \oplus J K^{\prime}$. It follows that $K=J K$. Since $K$ is locally projective, we get $K=0$, i.e., $h$ is a monomorphism. Thus $h$ is an isomorphism.

Recall that $R$ is semiperfect if $R / J$ is semisimple and idempotents lift modulo $J$. It is known that $R$ is semiperfect if and only if every simple (finitely generated, cyclic) left $R$-module has a projective cover (see, e.g. [AF, Theorem 27.6]). Azumaya [A2, Theorem 3] generalized this and proved that if every simple left $R$-module has a generalized projective cover then $R$ is semiperfect. Modifying his proof we generalize his theorem as follows.

Theorem 3.5. The following three statements are equivalent for a ring $R$.

(1) $R$ is semiperfect.

(2) Every simple left $R$-module has a locally projective cover.

(3) Every simple left $R$-module has a generalized locally projective cover.

Proof: $(1) \Rightarrow(2) \Rightarrow(3)$. These are clear.

(3) $\Rightarrow(1)$. To show $\bar{R}=R / J$ is semisimple, we only need to prove each simple left $\bar{R}$-module $S$ is locally projective (since a simple locally projective module is projective). We regard $S$ as a simple left $R$-module, so there is a generalized locally projective cover $f: P \longrightarrow S$, where $\operatorname{Ker}(f) \subseteq \operatorname{Rad}(P)=J P$. Since $\operatorname{Ker}(f)$ is a maximal submodule of $P$ we must have $\operatorname{Ker}(f)=J P$ and so $P / J P \cong S$. Since $P$ is a locally projective $R$-module, $P / J P$ is a locally projective $\bar{R}$-module, so $S$ is a locally projective $\bar{R}$-module. Therefore $\bar{R}$ is semisimple.

Let $\varepsilon$ be an idempotent of $\bar{R}$. We want to show that $\varepsilon$ can be lifted to an idempotent of $R$, so we may assume that $\varepsilon \neq \overline{0}$ and $\varepsilon \neq \overline{1}$. Then both $\bar{R} \varepsilon$ and $\bar{R}(\overline{1}-\varepsilon)$ are non-zero left ideals of the semisimple ring $\bar{R}$. Let $\bar{R} \varepsilon=S_{1} \oplus \cdots \oplus S_{k}$ and $\bar{R}(\overline{1}-\varepsilon)=S_{k+1} \oplus \cdots \oplus S_{n}$ be direct sums of simple left ideals $S_{i}$ 's. We view $S_{i}$ as a simple left $R$-module and let $f_{i}: P_{i} \longrightarrow S_{i}$ be a generalized locally projective cover $(i=1,2, \ldots, n)$. Then

$$
f=\oplus_{i=1}^{n} f_{i}: P=\oplus_{i=1}^{n} P_{i} \longrightarrow \oplus_{i=1}^{n} S_{i}
$$

is a generalized locally projective cover by Lemma 3.2 . Since the natural epimorphism $g: R \longrightarrow \bar{R}$ is a projective cover, by Proposition 3.4 there is an isomorphism $h: P \longrightarrow R$ such that $f=g h$. Let $L=h\left(P_{1} \oplus \cdots \oplus P_{k}\right)$ and $L^{\prime}=h\left(P_{k+1} \oplus \cdots \oplus P_{n}\right)$. Then $L$ and $L^{\prime}$ are left ideals of $R$ and 
$R=L \oplus L^{\prime}$. Let $L=R e$ and $L^{\prime}=R e^{\prime}$ for some idempotents $e$ and $e^{\prime}$ of $R$ with $e+e^{\prime}=1$. Let $\bar{e}=g(e) \in \bar{R}$. Then

$$
\begin{aligned}
\bar{R} \bar{e} & =g(R e)=g(L)=g h\left(P_{1} \oplus \cdots \oplus P_{k}\right)=f\left(P_{1} \oplus \cdots \oplus P_{k}\right) \\
& =f_{1}\left(P_{1}\right) \oplus \cdots \oplus f_{k}\left(P_{k}\right)=S_{1} \oplus \cdots \oplus S_{k}=\bar{R} \varepsilon
\end{aligned}
$$

Similarly, if we let $\bar{e}^{\prime}=g\left(e^{\prime}\right) \in \bar{R}$ then $\bar{R} \bar{e}^{\prime}=\bar{R}(\overline{1}-\varepsilon)$. Now $\overline{1}=g(1)=$ $g\left(e+e^{\prime}\right)=\bar{e}+\bar{e}^{\prime}$. By [AFF, Proposition 7.2] we must have $\varepsilon=\bar{e}$, i.e., $\varepsilon$ can be lifted to the idempotent $e \in R$.

Corollary 3.6. The following three statements are equivalent for a ring $R$.

(1) $R$ is semiperfect.

(2) Every finitely generated (cyclic) left $R$-module has a locally projective cover.

(3) Every finitely generated (cyclic) left $R$-module has a generalized locally projective cover.

Next we characterize semiperfect modules using locally projective covers, but we need a lemma first.

Lemma 3.7. If a module $M$ has a generalized locally projective cover then (1) $\operatorname{Rad}(M)=J M$; and (2) $M$ has a maximal submodule if $M \neq 0$.

Proof: Let $f: P \longrightarrow M$ be a generalized locally projective cover. Then $\operatorname{Ker}(f) \subseteq \operatorname{Rad}(P)=J P$. By [AF, Proposition 9.15], we have $\operatorname{Rad}(M)=f(\operatorname{Rad}(P))=f(J P)=J(f(P))=J M$. If $M \neq 0$, then $P \neq 0$ and $\operatorname{Rad}(P) \neq P$. So $P$ has a maximal submodule $U$. Since $\operatorname{Ker}(f) \subseteq \operatorname{Rad}(P) \subseteq U, f(U)$ must be a maximal submodule of $f(P)=$ $M$.

Proposition 3.8. A module $M$ is semiperfect if and only if $M$ has a projective cover and every factor module of $M$ has a locally projective cover.

Proof: $(\Rightarrow)$. This is obvious.

$(\Leftarrow)$. Let $U$ be a proper submodule of $M$. Then $M / U$ is a non-zero factor module of $M$. By Lemma 3.7, $M / U$ has a maximal submodule. This means that $U$ is contained in a maximal submodule of $M$. By assumption, every simple factor module of $M$ has a locally projective cover which is a projective cover by Lemma 3.3. Hence $M$ is semiperfect by $[\mathbf{A 2}$, Theorems 4 and 6$]$. 
Corollary 3.9. A projective module $M$ is semiperfect if and only if every factor module of $M$ has a locally projective cover.

Recall that $R$ is left perfect if every left $R$-module has a projective cover. An interesting characterization of left perfect rings was presented in $[\mathbf{A F}$, Theorem 28.4] which was due to Bass [B]. Now we characterize left perfect rings using (generalized) locally projective covers.

Theorem 3.10. The following three statements are equivalent for a ring $R$.

(1) $R$ is left perfect.

(2) Every left $R$-module has a locally projective cover.

(3) Every left $R$-module has a generalized locally projective cover.

Proof: $(1) \Rightarrow(2) \Rightarrow(3)$. These are clear.

$(3) \Rightarrow(1)$. By Theorem 3.5 or Corollary $3.6, R$ is semiperfect. By Lemma 3.7, every non-zero left $R$-module has a maximal submodule. Hence $R$ is left perfect by [AF, Theorem 28.4].

It is known that if every semisimple left $R$-module has a projective cover then $R$ is left perfect. We do not know whether the condition "projective cover" can be weakened to "locally projective cover".

Azumaya [A2, Theorem 1] showed that a flat module having a generalized projective cover is projective. An analogous result for locally projective modules is the following

Proposition 3.11. If $M$ is a flat module having a generalized locally projective cover then $M$ is locally projective.

Proof: Let $f: P \longrightarrow M$ be a generalized locally projective cover and $K=\operatorname{Ker}(f)$. Then $K \subseteq \operatorname{Rad}(P)=J P$. By [AF, Lemma 19.18] $K$ is a pure submodule of $P$, so $K$ is also locally projective and $J K=K \cap J P \supseteq$ $K$. We get $K=J K$, and so $K=0$. Hence $P \cong M$ and $M$ is locally projective.

As pointed out by Zimmermann-Huisgen in [Z2, p. 60], $R$ is left perfect if and only if every flat left $R$-module is locally projective. Hence by Proposition 3.11 we obtain

Corollary 3.12. A ring $R$ is left perfect if and only if every flat left $R$-module has a (generalized) locally projective cover.

Camillo and Xue $[\mathbf{C X}]$ called a ring $R$ left quasi-perfect in case every artinian left $R$-module has a projective cover, and showed that the class 
of left quasi-perfect rings lies strictly between that of left perfect rings and that of semiperfect rings. It was proved in $[\mathbf{C X}$, Theorem 1] that a semiperfect ring $R$ is left quasi-perfect if and only if every non-zero artinian left $R$-module has a maximal submodule (has finite length, is finitely generated). Now we characterize left quasi-perfect rings using (generalized) locally projective covers.

Theorem 3.13. The following three statements are equivalent for a ring $R$.

(1) $R$ is left quasi-perfect.

(2) Every artinian left $R$-module has a locally projective cover.

(3) Every artinian left $R$-module has a generalized locally projective cover.

Proof: $(1) \Rightarrow(2) \Rightarrow(3)$. These are clear.

$(3) \Rightarrow(1)$. Since simple modules are artinian, $R$ is semiperfect by Theorem 3.5. By (3) and Lemma 3.7, we see that every non-zero artinian left $R$-module has a maximal submodule. Hence $R$ is left quasi-perfect by $[\mathbf{C X}$, Theorem 1].

A module $M$ is called strongly artinian $[\mathbf{C X 2}]$ in case every proper submodule of $M$ has finite length. Clearly a strongly artinian module is artinian, but the converse is false. Cai and Xue [CX2, Theorem 4] proved that a semiperfect ring $R$ is left quasi-perfect if and only if every (non-zero) strongly artinian left $R$-module has a projective cover (has a maximal submodule). Using these results and modifying the proof of Theorem 3.13 we have our concluding result.

Theorem 3.14. The following three statements are equivalent for a ring $R$.

(1) $R$ is left quasi-perfect.

(2) Every strongly artinian left $R$-module has a locally projective cover.

(3) Every strongly artinian left $R$-module has a generalized locally projective cover.

Acknowledgements. The author wishes to thank Professor G. Azumaya for sending him the paper $[\mathbf{A 2}]$ which motivated the present work.

\section{References}

[AF] F. W. Anderson and K. R. Fuller, "Rings and Categories of Modules," 2nd. edition, Springer-Verlag, New York, 1992. 
[A1] G. Azumaya, Some characterizations of regular modules, Publ. Mat. 34 (1990), 241-248.

[A2] G. Azumaya, A characterization of semi-perfect rings and modules, in "Ring Theory," edited by S. K. Jain and S. T. Rizvi, Proc. Biennial Ohio-Denison Conf., May 1992, World Scientific Publ., Singapore, 1993, pp. 28-40.

[B] H. BAss, Finitistic dimension and a homological generalization of semiprimary rings, Trans. Amer. Math. Soc. 95 (1960), 466-488.

[CX] V. P. Camillo and W. Xue, On quasi-perfect rings, Comm. Algebra 19 (1991), 2841-2850; Addendum, 20 (1992), 1839-1840.

[CX2] Y. CAI AND W. Xue, Strongly noetherian modules and rings, Kobe J. Math. 9 (1992), 33-37.

[F] D. J. Fieldhouse, Semi-perfect and $F$-semi-perfect modules, $I n$ ternat. J. Math. Math. Sci. 8 (1985), 545-548.

[Z1] B. Zimmermann-Huisgen, Pure submodules of direct products of free modules, Math. Ann. 224 (1976), 233-245.

[Z2] B. Zimmermann-Huisgen, "Direct Products of Modules and Algebraic Compactness," Habilitationsschrift, Tech. Univ. Munich, 1980.

\author{
Department of Mathematics \\ Fujian Normal University \\ Fuzhou, Fujian 350007 \\ PEOPLE'S REPUBLIC OF CHINA
}

Rebut el 2 de Maig de 1995 